
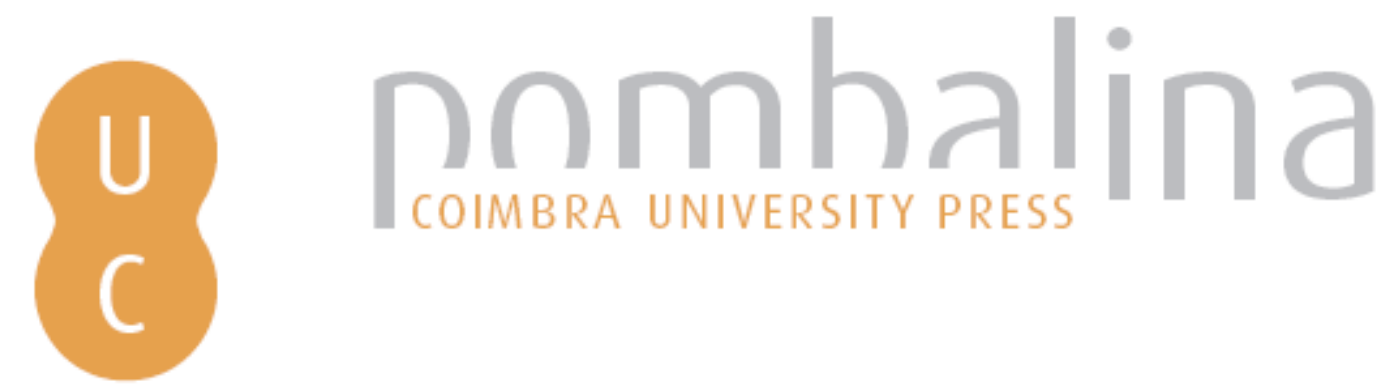

Desarrollo de tutoriales en Bibliotecas Universitarias en el contexto del aprendizaje y la investigación: adaptación al Espacio Europeo de Enseñanza Superior

Autor(es): $\quad$ Alonso Arévalo, Julio; Gallego Lorenzo, Josefa

Publicado por: Imprensa da Universidade de Coimbra

URL

persistente: URI:http://hdl.handle.net/10316.2/31908

DOI: $\quad$ DOI:http://dx.doi.org/10.14195/978-989-26-0319-3_25

Accessed : $\quad$ 26-Apr-2023 14:24:17

A navegação consulta e descarregamento dos títulos inseridos nas Bibliotecas Digitais UC Digitalis, UC Pombalina e UC Impactum, pressupõem a aceitação plena e sem reservas dos Termos e Condições de Uso destas Bibliotecas Digitais, disponíveis em https://digitalis.uc.pt/pt-pt/termos.

Conforme exposto nos referidos Termos e Condições de Uso, o descarregamento de títulos de acesso restrito requer uma licença válida de autorização devendo o utilizador aceder ao(s) documento(s) a partir de um endereço de IP da instituição detentora da supramencionada licença.

Ao utilizador é apenas permitido o descarregamento para uso pessoal, pelo que o emprego do(s) título(s) descarregado(s) para outro fim, designadamente comercial, carece de autorização do respetivo autor ou editor da obra.

Na medida em que todas as obras da UC Digitalis se encontram protegidas pelo Código do Direito de Autor e Direitos Conexos e demais legislação aplicável, toda a cópia, parcial ou total, deste documento, nos casos em que é legalmente admitida, deverá conter ou fazer-se acompanhar por este aviso.

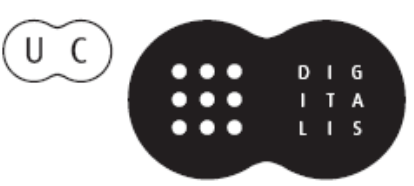


Maria Manuel Borges

Elias Sanz Casado

Coordenação

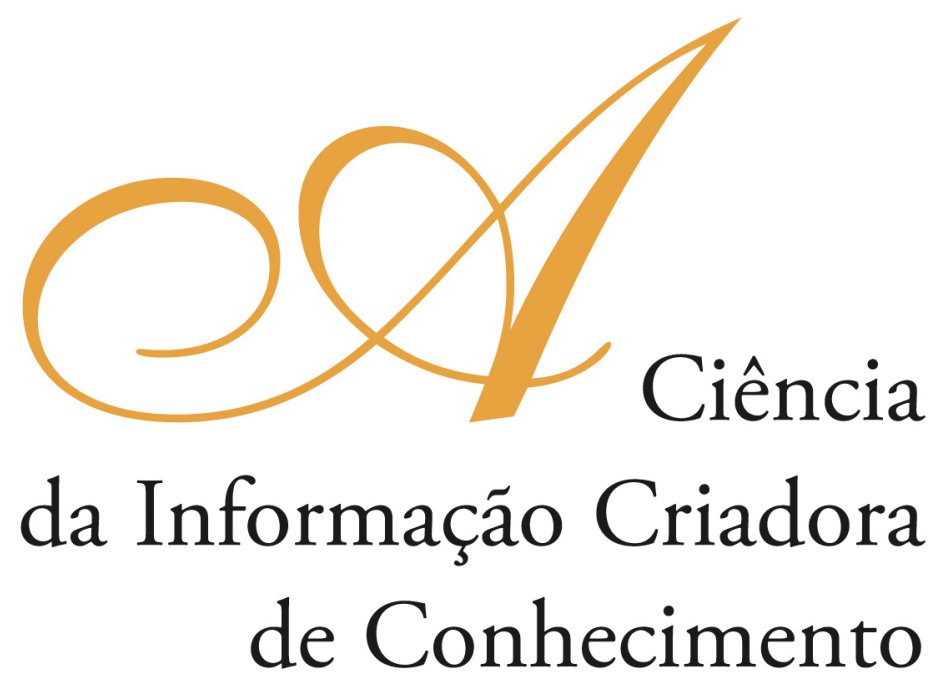

Vol. I

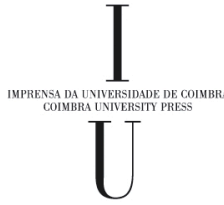

- COIMBRA 2009 


\title{
Desarrollo de tutoriales en Bibliotecas Universitarias en el contexto DEL APRENdizaje y La inVESTigación: adaptación al Espacio Europeo de Enseñanza Superior
}

\author{
Julio Alonso Arévalo \\ Universidad de Salamanca (Espanha) \\ Josefa Gallego Lorenzo \\ Universidad de León (Espanha)
}

\section{Resumen}

El nuevo contexto de aprendizaje e investigación que determina el Espacio Europeo de Enseñanza Superior, junto con un nuevo modelo de relación entre biblioteca-usuario que viene planteándose desde hace ya algunos años con las tecnologías de información, consagra la formación en información como una de las líneas estratégicas básicas en el futuro de los servicios bibliotecarios universitarios, y así lo reconoce el II Plan Estratégico REBIUN 2007-2010. Esto conlleva la implicación de la biblioteca en la elaboración de materiales de apoyo, y un esfuerzo del personal de los servicios de información para capacitarse adecuadamente. Hay que orientar la formación inicial y continuada en aspectos relativos a didáctica, usabilidad, y conocimiento de diversas herramientas, especialmente aquellas basadas en software libre como ExeLearning y CamStudio. Se presentan algunas experiencias iniciadas en este ámbito.

\begin{abstract}
The new context of learning and research determined by the European Higher Education Area, jointly with the new model of relationship between the library and its users that has been raising for some years with the information technologies, establishes training on information as one of the fundamental strategic lines in the future of academic library services, as recognized by REBIUN's II Strategic Plan 2007-2010. This implies the involvement of the library in the development of support materials, and an effort by the information services staff to be adequately trained. We must guide the education and training in aspects of teaching, usability, and knowledge of various tools, especially those based on free software as ExeLearning and CamStudio. Some experiments undertaken in this area are presented.
\end{abstract}

\section{Introducción}

El desarrollo de los formatos digitales unido a la generalización de las redes de información acaecida durante los últimos años han llevado a bibliotecas y servicios de información a replantearse diversas cuestiones relativas a temas de gestión de recursos, evaluación de colecciones, conductas de los usuarios en este nuevo entorno y sobre todo cómo responder eficaz y eficientemente al apoyo y capacitación de un nuevo tipo 
de usuario que acude a la biblioteca a través de impulsos de bits cómodamente desde su despacho o desde su domicilio 365 días al año y durante 24 horas diarias (Gómez Hernández; Benito Morales, 2001)

Quienes partimos hace ya algunos años del desarrollo de tareas profesionales de la biblioteca estrictamente presencial a la biblioteca digital en línea hemos sido afortunados testigos de un proceso de cambio operado en los últimos años, de dos formas diferentes de "hacer", de diferentes capacidades de llegar al usuario final, que aunque no han modificado la esencia de la biblioteca como entidad dentro del sistema de información y comunicación científica, sí han hecho replantearse nuevos retos y formas de trabajo para adaptarnos a la nueva situación.

La información digital ha planteado diferentes maneras de gestionar los recursos que abarcan desde la adquisición hasta su utilización por parte del usuario final. En esta primera fase de selección y adquisición se concreta un nuevo modelo distinto al tradicional que ha planteado nuevas formas de relación entre proveedores y gestores de información. La adquisición de recursos electrónicos ya no se hace de manera unitaria, es decir no nos suscribimos a una revista, si no que lo hacemos a un paquete de revistas que cubren las necesidades de información de nuestra comunidad de usuarios. Por otra parte no adquirimos la suscripción, si no que pagamos por una licencia de acceso cuyo precio no es tampoco unitario, y que en virtud de diferentes variables va a tener un precio final diferente, lo que conlleva a negociar los precios finales de las licencias de acceso con el distribuidor.

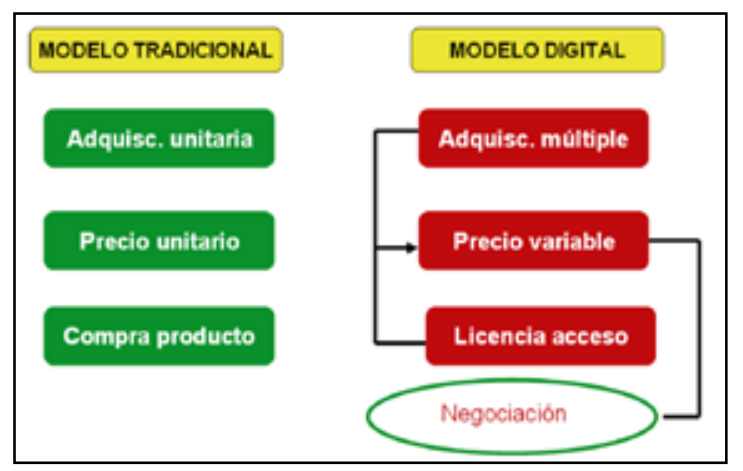

En lo relativo a la gestión de recursos también se han operado cambios que han afectado a nuestras formas de trabajo. Por una parte, el peso que se daba a los procesos técnicos que durante tanto tiempo fueron una función clave en el desarrollo profesional se ha visto minimizado a la localización del recurso en cualquier otro lugar y en la transferencia de los metadatos a través de sistemas tales como Z39.50.

Actualmente la percepción por parte del usuario de una biblioteca con un nivel de calidad aceptable es aquella que concreta sus esfuerzos en el desarrollo de servicios finales a los usuarios, entre los que estarían los relativos a apoyo y formación en el uso de información.

Sin embargo este desarrollo no solo ha afectado a la biblioteca, sino que también ha posibilitado plantearse nuevas formas de enseñanza y aprendizaje que con más o menos éxito se han ido concretando durante todos estos años. Cada vez estamos 
más familiarizados con ellas y es probable que bien como alumnos, o docentes hayamos participado en alguna ocasión en formatos de enseñanza en línea, formación semipresencial, o en el desarrollo de campus y entornos virtuales de aprendizaje.

Por otra parte en el plano político se ha buscado la compatibilización e integración de las enseńanzas superiores en el contexto europeo, en la propuesta conocida como Plan de Bolonia. Bolonia esencialmente pretende institucionalizar un nuevo sistema basado en el aprendizaje, que capacite al alumno para aprender investigando, en el que el peso de la clase magistral deja de ser el elemento primordial del sistema de enseńanza, para trabajar más como se ha venido haciendo en los países que han obtenido mejores resultados en lo relativo a la calidad de la formación a través de seminarios, en los que el profesor plantea el desarrollo de trabajos de campo, y en los que el alumno rastrea, recopila y contrasta información para la elaboración del mismo. Sobre todo se basa no tanto en transmitir conocimientos como en posibilitar metodologías y el trabajo con fuentes de información. Parece lógico que en esta nueva situación la biblioteca tenga un papel también más activo, como de hecho ya ocurre en otros países donde el bibliotecario es librarianship, pero también es scholarship, es decir que parte de su tarea profesional es formar a la comunidad académica en el uso más eficaz de los recursos de información.

Como no podría ser de otra manera, la alfabetización digital es una de las líneas prioritarias de actuación de REBIUN (Red de Bibliotecas Universitarias de España). Las bibliotecas y servicios de información científica están trabajando en este sentido, aunque con diferentes niveles de desarrollo. Cada vez con más frecuencia encontramos que las diferentes sedes web de las bibliotecas universitarias españoles integran recursos de apoyo a la formación en información a través de diferentes medios: chat, correo electrónico, cursos presenciales, tutoriales, redes sociales, etc.

En el nuevo contexto de Bolonia las bibliotecas tendrán que asumir nuevas funciones y redefinir sus objetivos; hay quienes han propuesto incluso un cambio de denominación hacia Centros de Recursos para el Aprendizaje (CRAI). Probablemente el cambio de nombre sea lo de menos, la biblioteca se ha ido adaptando a los diferentes entornos y a las circunstancias que a lo largo de su dilatado recorrido han ido planteándose, pero sí parece clave que la formación en información va a cobrar un peso considerable, como pone de manifiesto Ferrán Ferrer $(2007,15)$ "En este contexto se dibujan los retos para el nuevo papel de las bibliotecas como garantes y facilitadoras de estos nuevos entornos de aprendizaje".

\section{Alfabetización digital}

La alfabetización digital comprende diversas líneas de actuación: por una parte una formación directa como la que se ejerce en el espacio de la biblioteca, muchas veces en el propio día a día, y que es de una efectividad absoluta ya que habitualmente va dirigida a la solución de un problema concreto planteado por un usuario "in situ". Podríamos decir que se trata de una prolongación del sistema de información y referencia de la biblioteca, que resuelve un problema o necesidad informativa en el momento que éste se le plantea al usuario. Por otra parte existen otras actividades directas encaminadas tanto a la promoción de la biblioteca y sus servicios, como a la orientación del usuario en la misma: se trata de actividades de formación en información tanto en el aula como 
en la propia biblioteca. Este tipo de sesiones tienen éxito cuando están regladas, y se asumen como una necesidad que va a tener el nuevo alumno en la universidad, y por lo tanto cuenta con el apoyo y la participación activa del equipo de dirección del centros y con la colaboración de los propios docentes que se implican en ceder un espacio de su tiempo a estas actividades. Durante estos años ya algunas universidades han asumido la inclusión en los planes de estudio una formación instrumental en muchos casos impartida por personal bibliotecario que capacite a la comunidad universitaria en el manejo de las herramientas de aprendizaje (Gómez Hernández, 2000).

Estas actividades pueden estar encaminadas a la formación inicial en el momento en que el alumno llega a la universidad. Según nuestra propia experiencia de más de 18 , esta primera toma de contacto debe ir encaminada a ser un punto de encuentro con la biblioteca, el personal y los servicios más que orientarse a ser una actividad formativa intensa sobre recursos y servicios. Posteriormente a medida que el alumno tiene necesidades de información más específicas se propondrán otras acciones directas más concretas que podrían coincidir con su entrada en un segundo ciclo, o formación de master, tales como trabajo directo con recursos de información digital especializados, gestores de referencias y herramientas de trabajo.

\section{Objetos y plataformas de aprendizaje}

Sin menoscabo de la formación presencial y directa, en este contexto cada vez van a cobrar más importancia el apoyo mediante plataformas de aprendizaje en línea y la elaboración de objetos de aprendizaje.

Casi todas las universidades se han planteado y han desarrollado plataformas para el aprendizaje en el contexto de lo que se ha denominado e-learning o enseńanza virtual, tanto como apoyo a las actividades presenciales, como en la elaboración de proyectos de enseñanza semipresencial o en línea. Estas plataformas en las instituciones públicas se han realizado con software libre como Moodle, por tres cuestiones fundamentales: de una parte, un decidido y firme apoyo al software de código abierto por parte de las administraciones públicas, que las hace menos dependientes de las multinacionales del software; y de otra, porque posibilita desarrollos muy rápidos ya que son muchas las personas que trabajan voluntariamente para optimizar estos productos, y por último por el ahorro en la adquisición de licencias.

IEEE considera que los objetos de aprendizaje son una entidad que puede ser utilizada, reutilizada y referenciada durante el aprendizaje en donde se utiliza la tecnología como medio de transmisión de conocimiento. Es decir un objeto digital que puede ser reutilizado como apoyo al aprendizaje.

Como ejemplos de objetos de aprendizaje podemos encontrar aquellos que se basan en contenidos multimedia, software para el aprendizaje, organizaciones, eventos referenciados durante el aprendizaje basado en tecnología, o bien objetos más simples como archivos de texto, ilustraciones, vídeos o fotografías ${ }^{1}$.

\footnotetext{
${ }^{1}$ Universitat Politècnica de Catalunya (UPC). Servei de Biblioteques i Documentació (2008). Repositorios docentes. Aspectos a considerar en su implementación. Madrid: Rebiun
} 
La importancia de los objetos de aprendizaje empieza a ser tal que buena parte de los repositorios institucionales de acceso abierto lo empiezan a considerar como un material objeto de ser recogido en esa memoria institucional que es el repositorio.

\section{Tutoriales}

Un tutorial es una guía o recorrido que tiene un fin educativo a través de pasos explicativos con una alta capacidad comprensiva para facilitar la utilización y manejo de un recurso o servicio.

Por medio de los tutoriales un usuario puede aprender de manera independiente la utilización de un servicio, la organización y reglamentación del mismo u otras acciones que tienen por objetivo el desarrollo de habilidades que les conviertan en usuarios autónomos.

Los tutoriales pueden integrar imágenes, sonidos, animaciones, secuencias dinámicas online, etc.

Las características que debe tener un buen tutorial son:

- Impacto visual

- Usabilidad (Mackey; Ho, 2008)

- Entorno remoto

- Interactividad

- Adaptabilidad

- Ser eficientes, dinámicos y atractivos

Nancy Dewald (1999) establece siete indicadores fundamentales que debe tener un tutorial para ser eficaz: estar directamente relacionado con el contenido de la asignatura que apoya, fomentar el aprendizaje activo, estimular el aprendizaje colaborativo, ofrecerse en más de un medio, tener el objetivo de enseñar e incluir la posibilidad de contacto o consulta al bibliotecario si se necesita ayuda.

Kornelia Tancheva (2003) señala que un tutorial no sólo debe contener elementos audiovisuales, animación y gráficos, sino que debe ser interactivo y modular, ser capaz de evaluar los conocimientos previos del alumno, enseñar los conceptos, estar vinculado a una disciplina específica, prestar asesoramiento, y registrar las actividades del estudiante durante las sesiones.

\section{Herramientas para la elaboración de tutoriales}

Algunas bibliotecas utilizan formatos comunes para elaborar tutoriales de aprendizaje tales como documentos en Word, PDF o presentaciones generadas en Power Point (Campal García, 2004), pero existen herramientas específicas muchas de ellas de software libre o freeware ${ }^{2}$ y del entorno 2.0 que nos pueden ser de gran ayuda para elaborar productos más profesionales.

\footnotetext{
${ }^{2}$ Aplicación informática que se puede copiar y distribuir libremente, y cuyo uso es gratis. Aunque si tiene licencia y no es software libre de código abierto.
} 


\section{Generadores de objetos de aprendizaje}

\section{1. eXelearning}

eXeLearning ${ }^{3}$ es un programa creado por la Auckland University of Technology y el Tairawhiti Polytechnic para crear recursos educativos de una forma sencilla e intuitiva sin precisar grandes conocimientos técnicos. eXeLearning genera sus contenidos en páginas web o paquetes comprimidos IMS, SCORM 1.2 o COmmon Cartridge. Es un software gratuito y abierto. eXeLearning permite crear un tutorial web con una página con un menú lateral dináminco, así como editar páginas con contenido multimedia, personalizar el tutorial por medio de diferentes hojas de estilo, y exportar como sitio web ${ }^{4}$.

\section{EdLim}

Lim es un entorno para la creación de materiales educativos formado por un editor de actividades (EdiLim), un visualizador (LIM) y un archivo en formato XML (libro) que define las propiedades del libro y las páginas que lo componen.

Ventajas:

- No es necesario instalar nada en el ordenador.

- Accesibilidad inmediata desde internet.

- Independiente del sistema operativo, hardware y navegador web.

- Tecnología Macromedia Flash, de contrastada fiabilidad y seguridad.

- Entorno abierto, basado en el formato XML.

Desde el punto de vista educativo:

- Entorno agradable.

- Facilidad de uso para los alumnos y el profesorado.

- Actividades atractivas.

- Posibilidad de control de progresos.

- Evaluación de los ejercicios.

- No hay que preparar los ordenadores, es un recurso fácil de manejar.

- Posibilidad de utilización con ordenadores, PDAs y pizarras digitales interactivas.

- Creación de actividades de forma sencilla.

LIM precisa para su funcionamento el plug-in Flash. Podemos ver un tutorial sobre EdLim en la web de la Universidad de Salamanca ${ }^{5}$ creado con EdLim

3 eXeLearning. http://wiki.exe-spain.es/doku.php

${ }^{4}$ Poveda, A. Construir lecciones virtuales con Exelearning. Tutorial del Servicio de Bibliotecas de la Universidad de Salamanca http://web.usal.es/-angelpoveda/web\%20biologia/tutoriales/Construir\%20lecc iones\%20virtuales\%20con\%20Exelearning/index.html

${ }^{5}$ Poveda, A. (2009). EdiLIM: generar ejercicios de uso docente. Salamanca: Universidad de Salamacna, Servicio de Bibliotecas. Disponible en: http://web.usal.es/-angelpoveda/web\%20biologia/tutoriales/ EDILIM/index.html 
Flash (. SWF)

El formato Flash incluye vídeo de movimiento completo de la actividad de escritorio, -movimiento del cursor y escritura- al que se puede sincronizar con el audio de la narración del instructor, y etiquetas en un formato de archivo de Flash (. SWF) que es accesible a través de la Web.

Este tipo de animación puede realizarse a través de diferentes programas que primero graban las acciones directamente del escritorio en un formato de video. AVI, y que posteriormente comprimen a SWF, pudiendo quedar la animación con un peso inferior a 3 megabytes, es decir con tamaño para ser utilizado a través de la web, al que podemos ańadir elementos tales como audio sincronizado y etiquetas explicativas.

En el ámbito del software propietario un programa que realiza estas acciones de manera sencilla y fácil es Adobe Captivate ${ }^{6}$. Con Adobe Captivate se pueden grabar en secuencias de vídeo toda la actividad desarrollada en la pantalla del ordenador. Todo movimiento de cursor, programa que se ejecute o lo que se escriba queda registrado en un video que se puede visualizar o utilizar para una presentación. Además en su versión 4 tiene capacidad de crear una voz en off automática que transforma el texto en voz de alta calidad. También incluye widgets que permite incluir cuestionarios de preguntas en la presentación. Las presentaciones se pueden grabar en formato Flash y se le puede añadir interactividad sin necesidad de tener conocimientos ni de Flash ni de programación. Se puede descargar una versión demo en el enlace: http://adobecaptivate.softonic.com/

También se dispone de programas similares en software libre y freeware.

En software libre y código abierto se dispone de CamStudio ${ }^{7}$, progrma que permite fácilmente grabar la actividad de la pantalla en una película de vídeo en formato AVI o SWF (Flash). Cualquier acción como mover el cursor del ratón, hacer clic, o escribir caracteres es registrado por el programa y se puede visualizar más tarde como una película. Este programa puede ser útil para demostraciones de utilización de software o grabar secuencias de una película en reproducción. Es de gran utilidad para elaborar tutoriales. Se puede descargar gratis.

En freeware, aunque no es software libre, ni esta disponible en código abierto tenemos la alternativa Powerpoint to Flash, un conversor de archivos Power Point a Flash. Su funcionamiento es muy simple: se debe añadir la presentación de PowerPoint arrastrándola a la ventana del programa "PowerPoint to Flash", pulsar en Convert y se obtiene el documento en formato Flash apto para elaborar un tutorial.

Otra opción es Wink freeware que permite realizar presentaciones y tutoriales en formato flash, y posibilita ańadir comentarios y etiquetas a la misma. Para hacer una presentación con $\mathrm{Wink}^{8}$, se ejecuta el software objetivo de la presentación y se van capturando pantallas de las acciones que se quieren mostrar. Posteriormente Wink unirá cada una de estas pantallas en una película continua. La edición de la película permitirá ańadir o eliminar pantallas (también llamadas «marcos» en la aplicación) en

${ }^{6}$ Adobe Captivate. http://www.adobe.com/es/products/captivate/

7 CamStudio. http://camstudio.org/

${ }^{8}$ Wink. http://www.debugmode.com/wink/download.php 
cualquier punto, añadir comentarios, botones de anterior, siguiente o incluso saltar de un marco a otro. Trabaja tanto en entorno Windows como en Linux.

\section{Pantallas}

Amor Screen Capturo es una herramienta que permite realizar capturas de pantalla en un rectángulo en el área que el usuario desee, pudiendo guardarlas en los formatos más conocidos, como por ejemplo BMP, PNG, PCX, TIF, GIF o JPG.

Amor Screen Capture tiene la ventaja de que puede capturar la pantalla completa, una ventana específica de un programa, menús de programa, pantallas de MSDOS, fuentes DirectX, ventanas deslizantes, imágenes de cámaras digitales y, en definitiva, cualquier actividad que se produzca en el escritorio del ordenador. Amor Screen Capture es muy sencillo de usar, y trabaja a gran velocidad, permitiendo editar e imprimir las imágenes capturadas. Ideal para la elaboración de tutoriales.

\section{Web 2.0}

Algunos de los recursos de la denominada web 2.0 también son útiles en la elaboración de tutoriales que se pueden insertar posteriormente en una web (Gértrudix Barrio et al, 2007).

El más conocido es YouTube en el cual podemos colgar o subir un vídeo tutorial para luego incluirlo en nuestro sitio web (Mastromatteo, 2008). Son muchas las bibliotecas que ya disponen de vídeo que utilizan tanto para visitas guiadas como para servir de herramienta instrumental en apoyo a la utilización de un programa o recurso. Hay que decir que a veces la calidad del producto no es la deseada, ya que realizar un vídeo requiere un buen equipo de producción del que a veces no dispone la biblioteca.

SlideShare ${ }^{9}$ el sitio de Internet en el que se pueden subir presentaciones en formato PPS o PPT puede ser también una herramienta útil para insertar presentaciones en formato flash encastradas en código $\operatorname{HTML}($ Embebed) en las páginas de nuestra biblioteca con tutoriales. Para ello es necesario registrarse en SlideShare y alojar la presentación; posteriormente se crea una animación en Flash que podemos incluir en nuestra web.

Second Life (López Hernández, 2008) es un entorno virtual en tres dimensiones que muchos consideran simplemente un juego pero que tiene enormes posibilidades para la enseñanza a distancia y la comunicación. Algunas bibliotecas universitarias están intentando aprovecharlo en este sentido; la Universidad Carlos III es una de ellas.

Otra herramienta 2.0 que puede ayudar en la creación de elementos interactivos es Issuu ${ }^{10}$ que es una biblioteca en línea comunitaria. Una vez registrados, se suben páginas en PDF y el sistema las convierte a formato FLASH, creando una publicación virtual en línea, donde podemos pasar las páginas con la sensación de estar leyendo una revista. Dispone de un modo de pantalla completa, aunque tiene una limitación máxima a $100 \mathrm{MB}$ y 500 páginas. Además tal como ocurre con otras herramientas 2.0, permite que otras personas inserten sus comentarios, incluir como favoritos o

\footnotetext{
9 Slideshare http://www.slideshare.net/

${ }^{10}$ Issuu. http://issuu.com/
} 
compartir en redes sociales. Esta última opción es muy interesante, pues podemos insertarlos en nuestros blogs y utilizarlos para nuestros tutoriales o para el boletín de novedades ${ }^{11}$ de nuestra biblioteca.

\section{Audio y podcast}

El término podcast surge de las palabras iPod y broadcast (difusión). Consiste en la creación de archivos de sonido (generalmente en formato mp3 o AAC, y en algunos casos el formato libre ogg) o de vídeo (llamados videocasts o vodcasts) y su distribución mediante un sistema de sindicación que permita suscribirse (por ej. RSS) y usar un programa que lo descarga de Internet para que el usuario lo escuche en el momento que quiera, generalmente en un reproductor portátil ${ }^{12}$. A partir de este formato podemos crear también tutoriales de apoyo que los propios estudiantes pueden bajarse a sus reproductores Mpeg o Mp4 personales para escuchar de manera independiente. Parece necesario adaptarse a los tiempos y buscar puntos y elementos de encuentro con los estudiantes en aquellos medios en los que ellos se desenvuelven con más soltura. Una página interesante en este sentido es Wiki de podcast-es, http://www. podcast-es.org/index.php/Portada donde hay disponible un gran listado de podcasts en castellano y recursos sobre cómo hacerlos y cómo escucharlos. También hay podcasts en otros idiomas, euskera, catalán o gallego.

Otras páginas como Loquendo proporcionan un demo para generar a partir de texto escrito mensajes de voz que se pueden utilizar en la elaboración de tutoriales. Dispone de emulaciones de voz en diferentes idiomas y géneros (masculina, femenina). Simplemente hay escribir el texto, pulsar PLAY y oir o guardar un documento en WAV. El máximo permitido en esta demo son 500 caracteres. En Universo Abierto ${ }^{13}$ se dispone de un ejemplo.

NetVoice ${ }^{14}$ el proyecto Net4Voice pretende incrementar la calidad de las oportunidades al promover la adopción de entornos de aprendizaje sin barreras y desarrollar metodologías innovadoras que utilizan tecnologías de reconocimiento del habla. Estas últimas pueden transformar automáticamente el discurso de un conferenciante en un texto digital en tiempo real, generando una transcripción electrónica de la lección o conferencia lista para imprimirse o distribuirse mediante distintos dispositivos y canales. El proyecto Net4Voice, que empezó en diciembre de 2007 y termina en noviembre de 2009, asocia la participación de tres universidades y dos institutos de educación secundaria, donde estudiantes con distintos tipos de discapacidades auditivas o motoras pueden beneficiarse del uso de las tecnologías de reconocimiento del habla para tomar cursos en un entorno de aprendizaje accesible sin apoyo intermediario suplementario.

\footnotetext{
${ }^{11}$ Boletin de Novedades de la Biblioteca Pública Municipal Torrente Ballester de Salamanca http://issuu. com/videotecatorrenteballester/docs/novedades_videoteca_julio_09/3

12 Wikipedia "Podcasting". http://es.wikipedia.org/wiki/Podcasting

13 Universo Abierto. Blog de la Biblioteca de la Facultad de Traducción y Documentación de la Universidad de Salamanca. http://www.universoabierto.com/917/acceso-remoto-a-los-recursos-electronicos-de-la-usal/

${ }_{14}$ Net4Voice. https://www.net4voice.eu/net4voice/default.aspx
} 
Los que estudian una segunda lengua también se benefician de estas tecnologías ya que, una vez que ha concluido la clase, pueden acceder a las transcripciones multimedia disponibles y leer o escuchar la clase nuevamente.

\section{Situación española: Rebiun y análisis de las webs de las bibliotecas universitarias en la integración de recursos y objetos de aprendizaje.}

\section{Contexto y nueva situación y desarrollo tecnológico}

La llegada del Espacio Europeo de Educación Superior (EEES) no sólo va a suponer la posibilidad de compatibilizar las titulaciones en el ámbito comunitario, si no también el momento clave para introducir mejoras docentes (Vives-Gracia, 2004). Las bibliotecas deben contribuir conjuntamente cooperando con los docentes en la consecución de estos objetivos que hagan de los alumnos personas autónomas en el uso de la información.

Entre los esfuerzos desarrollados por diferentes bibliotecas universitarias españolas destacamos algunas buenas prácticas en la elaboración de tutoriales de apoyo (SomozaFernández; Abadal, 2009).

El II Plan Estratégico de REBIUN 2007-2010 ${ }^{15}$ establece en su línea 2a $2^{\text {a }}$ Objetivo estratégico: Habilidades en Información (Programa ALFIN) la necesidad de potenciar y promover acciones para el desarrollo e implementación de las Habilidades en Información como competencias transversales en el nuevo modelo docente:

"coordinar y crear proyectos conjuntos relacionados con la comunicación y difusión de formación virtual (asignaturas virtuales, campus virtual, etc.), materiales y tutoriales (guías, orientaciones, programas, etc.) relacionados con la formación y aprendizaje de usuarios en ALFIN. Incluir proyectos de formación a bibliotecarios en materia de docencia no presencial".

Las estadísticas de Rebiun en su punto 2.7.3 y 2.7.4 recogen entre sus datos el número de tutoriales empleados por los usuarios y el número de descargas de tutoriales ${ }^{16}$. Tutoriales en BU españolas

En este apartado recogemos páginas de bibliotecas universitarias españolas donde podemos encontrar buenas prácticas en la creación de tutoriales. Fernández Somoza (2009) en su tesis sobre el análisis de tutoriales en Bibliotecas Universitarias españolas, sostiene que la mayoría de los tutoriales han sido tratados en el contexto de las bibliotecas académicas, según la autora, se centran principalmente en explicar el desarrollo de la aplicación en un entorno concreto y sus principales características, pero pocos de ellos presentan aspectos que transcienda a una visión más general Según los datos recogidos en este trabajo sólo diecisiete centros, es decir el $24 \%$ han elaborado algún material formativo propio, la temática está muy relaciona con recursos concretos o la difusión de los servicios, fundamentalmente se centran en materiales instrumentales;

\footnotetext{
15 II Plan Estratégico de REBIUN 2007-2010. http://www.rebiun.org/export/docReb/plan.pdf

${ }^{16}$ REBIUN. Estadísticas. http://estadisticas.rebiun.org/cuestionarios/indicadores/indicadores_main.asp
} 
una pequeña parte de ellos incorporan ejercicios para que el alumno pueda evaluar su aprendizaje. y sólo un tercio dispone de la posibilidad de apoyo personalizado en línea. Las herramientas que se utilizan para su elaboración y difusión pública son fundamentalmente presentaciones en Power Point aproximadamente la mitad de los casos, archivos en PDF en un 20\%, archivos en vídeo Flash en un $20 \%$ y el resto en formato HTML (Adobe Captative y eXeLearning).

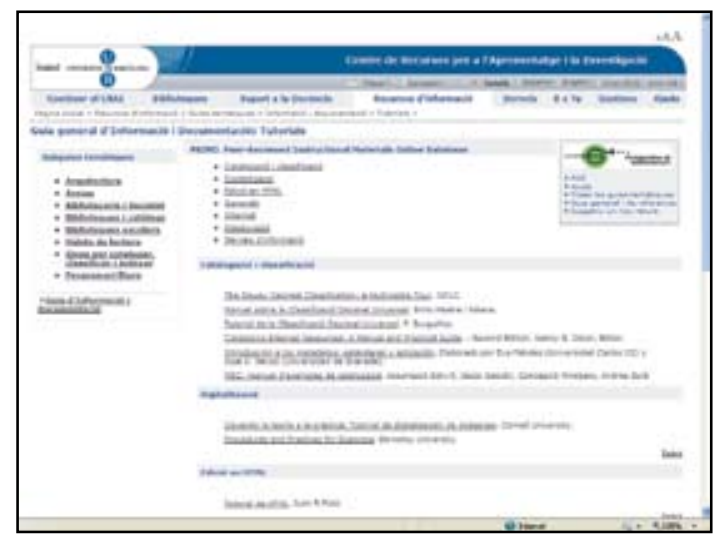

Biblioteca de la Universitat de Barcelona. Centre de Recursos per a l'Aprenentatge i la Investigació. Guia general d'Informació i Documentació: Tutorials http://www.bib.ub.edu/recursos-informacio/guies-tematiques/informaciodocumentacio/guia/tutorials/

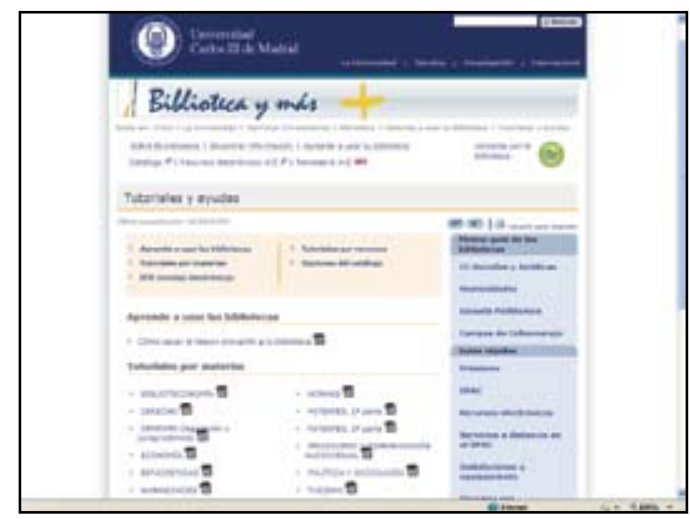

Biblioteca Universidad Carlos III. Tutoriales por materias. http://www.uc3m. es/portal/portal/page/portal/biblioteca/aprende_usar/tutoriales_y_ayudas\#aprende 


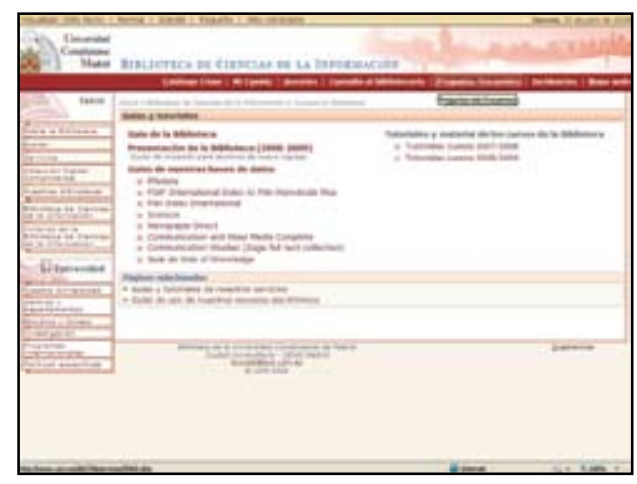

Biblioteca de la Universidad Complutense de Madrid. Tutorial de la Biblioteca. http://www.ucm.es/BUCM/inf/10426.php

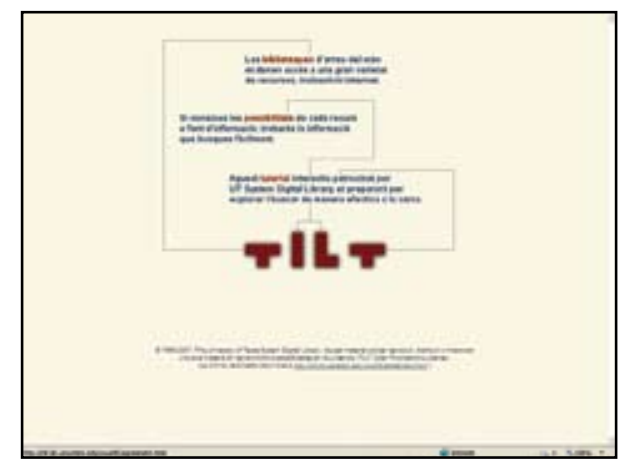

Biblioteca de la Universitat Politècnica de Catalunya.TILT. http://bibliotecnica. upc.es/tilt/

Se ha traducido al catalán el tutorial de código abierto que ofrece la universidad de Tejas TILT - The Information Literacy Tutorial para aquellas bibliotecas que deseen utilizarlo y adaptarlo a sus necesidades específicas

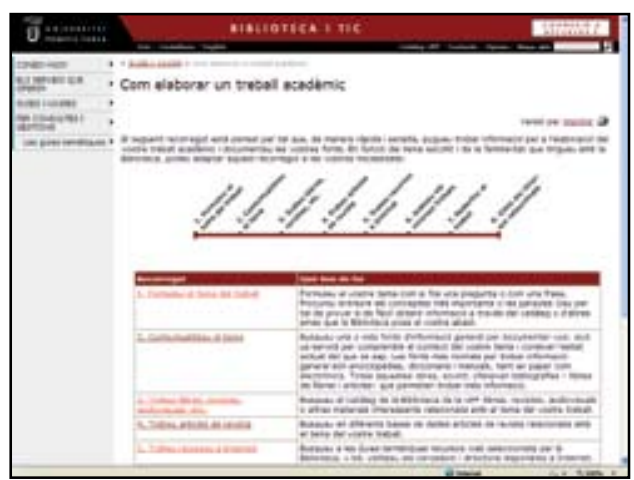

Universitat Pompeu Fabra. Com elaborar un treball acadèmic. http://www.upf. edu/bibtic/guiesiajudes/tre-aca.html 


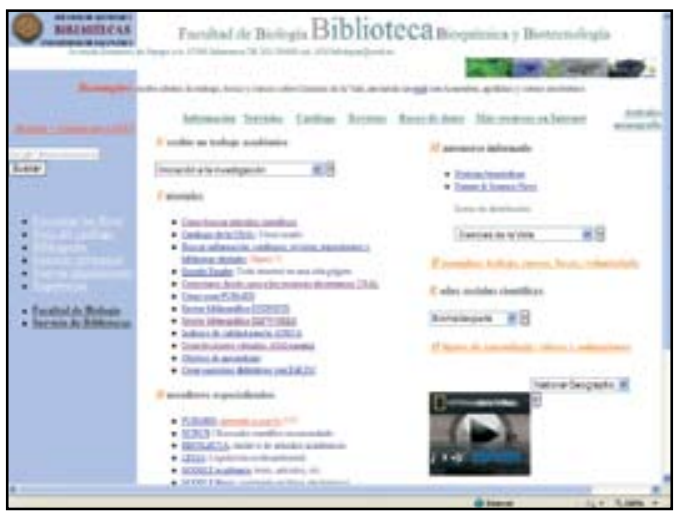

Biblioteca de Biología. Universidad de Salamanca. Tutoriales http://web.usal. es/-angelpovedalweb\%20biologial

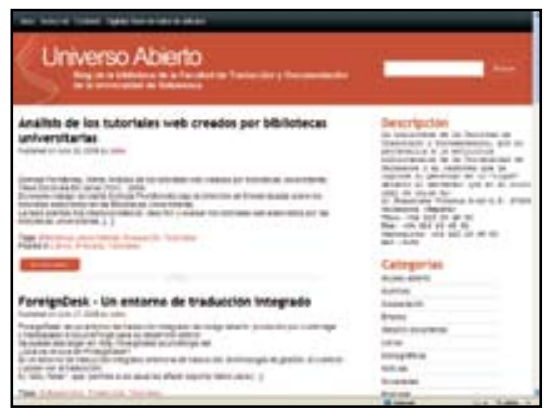

Universidad de Salamanca. Blog Universo Abierto. Tutoriales http://www. universoabierto.com/tag/tutoriales/

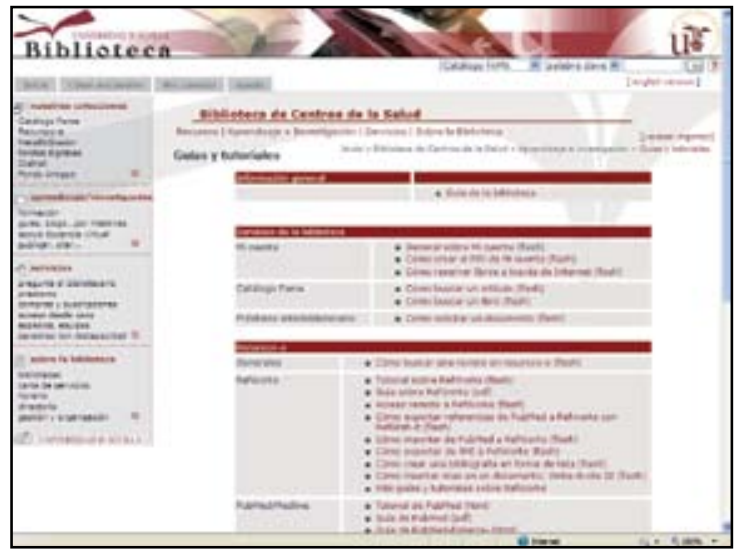

Biblioteca de la Universidad de Sevilla. Guias y tutoriales. http://bib.us.es/salud/aprendizaje_investigacion/guias_tutoriales-ides-idweb.html 


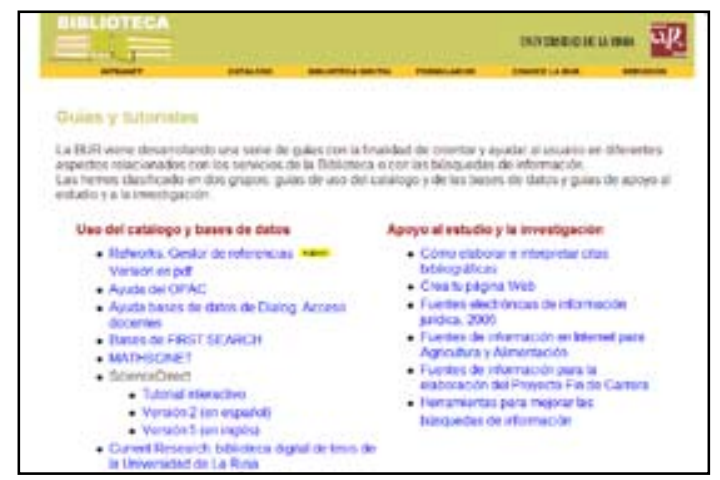

Biblioteca de la Universidad de la Rioja. Guías y tutoriales. http://biblioteca.unirioja.es/bdg/bdgguias.html

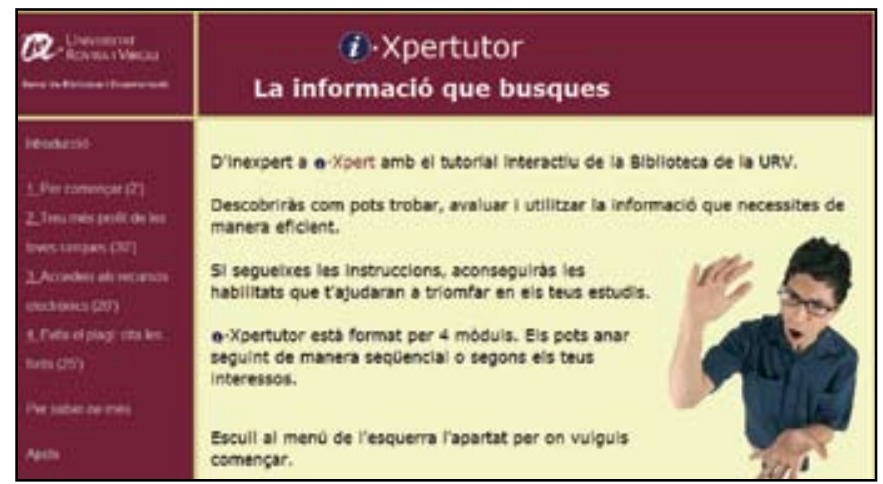

Documentació de la Universitat Rovira i Virgili. Tutorial i. Xpertutor http://www.urv.es/man/tutorial/

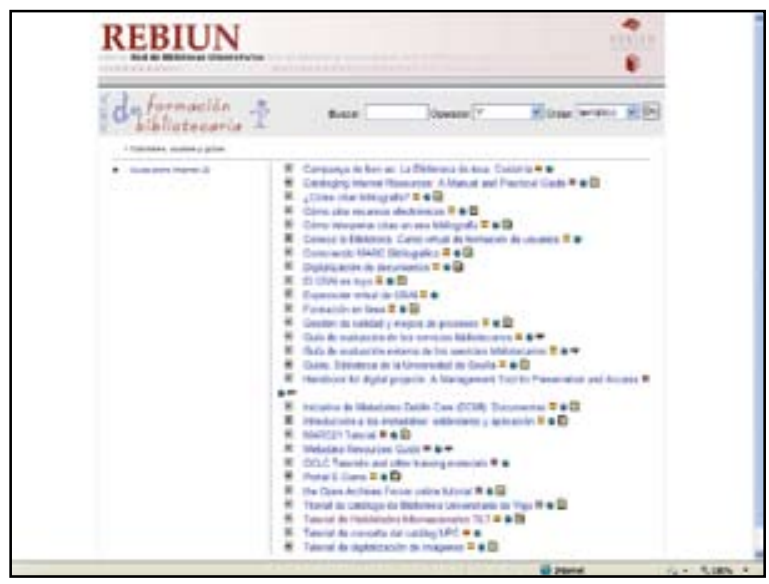

Rebiun. Portal de formación bibliotecaria.

http://aps.unirioja.es/biblio/recursos?cod $1=$ D\&dir $=$ tipo\&sub $=2$ 


\section{Referencias bibliográficas}

Alonso Arévalo, J. (2009). Acceso remoto a los recursos electrónicos de la USAL En Universo Abierto. Blog de la Biblioteca de la Facultad de Traducción y Documentación de la Universidad de Salamanca. Disponible: http://www.universoabierto.com/917/acceso-remotoa-los-recursos-electronicos-de-la-usal/

Bailin, A.; Peña, A. (2007). Online Library Tutorials, Narratives, and Scripts. The Journal of Academic Librarianship, 33, 1, 106-117. Disponible en: http://www.sciencedirect.com/ science/article/B6W50-4MM2621-1/2/ed16b1533a2721914022662cbe5d40de

Beile, P. M.; Boote, D. N. (2004). Does the medium matter?: A comparison of a Web-based tutorial with face-to-face library instruction on education students' self-efficacy levels and learning outcomes. Research Strategies, 20, 1-2, 57-68. Disponible en: http://www. sciencedirect.com/science/article/B6W60-4H6GPN0-2/2/65d2a998d508ead59d557687 c00194b6

Campal García, M. F.; González Palacios, L. A.; Cebrián Domínguez, I.; Martín Martín, M. (2004). Cartilla práctica para un tutorial de uso en las bibliotecas públicas. AABADOM, 2, 15, 14-20. http://www.aabadom.org/2004_1_2_cartilla.pdf

Campal García, M. F. (2004). Prácticas y experiencias de alfabetización informacional a través de tutoriales-web en las bibliotecas. En $V$ Coloquio Internacional de Ciencias de la Documentación. Universidad de Salamanca. Disponible en: http://web.usal.es/-fcampal/ investigacion/ExperienciasTutoriales.pdf

Carrozzo, M.; Lacquaniti, F. (1998). Virtual reality: a tutorial. Electroencephalography and Clinical Neurophysiology/Electromyography and Motor Control, 109, 1, 1-9. Disponible en: http://www.sciencedirect.com/science/article/B6T00-3S1PFT6-1/2/ f7c2d2a6bb4d2dbffc1 eadfa49c9b312

Churkovich, M.; Oughtred, C. (2002). Can an online tutorial pass the test for library instruction? An evaluation and comparison of library skills instruction methods for first year students at Deakin University». AARL. Australian Academic \& Research Libraries , 33, 1, Disponible en: http://alia.org.au/publishing/aarl/33.1/full.text/churkovich.oughtred.html

Dewald, N. H. (1999). "Transporting good library instruction practices into the web environment: An analysis of online tutorials». The Journal of Academic Librarianship, 25, 1, 26-31. Disponible en: http://www.sciencedirect.com/science/article/B6W50-3YCDKSR5/2/ac3ac9caa60be42ae9e3e41ac9a2dc2b

Donaldson, K. A. (2000). Library Research Success: Designing an Online Tutorial to Teach Information Literacy Skills to First-Year Students. The Internet and Higher Education, 2, 4, 2000, 237-251. Disponible en: http://www.sciencedirect.com/science/article/B6W4X423HJY3-5/2/0d0172d76bfc94e1e6307581e075c031

Ferrán Ferrer, N.; Minguillón Alfonso, J. (2007). El papel de las bibliotecas digitales en el acceso a los recursos educativos abiertos. En X Jornadas Españolas de Documentación, 287293. Disponible en: http://personal.uoc.edu/personalonto/files/Fesabid_nferranf.ppt

Gértrudix Barrio, M.; Alvarez, S., Galisteo, A.; Gálvez de la Cuesta, M. C.; Gértrudix Barrio, F. (2007). Acciones de diseño y desarrollo de objetos educativos digitales: programas institucionales. Revista de Universidad y Sociedad del Conocimiento, 4, 1. Disponible en: http://www.uoc.edu/rusc/4/1/dt/esp/gertrudix_alvarez_galisteo_galvez.pdf

Gómez Hernández, J. A. (2000). Prácticas y experiencias de alfabetización informacional en universidades españolas. En Congreso Internacional sobre Retos de la Alfabetización 
tecnológica en un mundo en red, 1. Disponible en: http://www.um.es/gtiweb/jgomez/hei/ intranet/heipresentaciontema5.ppt\#3

Gómez Hernández, J. A.; Benito Morales, F. (2001). De la formación de usuarios a la alfabetización informacional: propuestas para enseñar las habilidades de información. Scire, Representación y Organización del Conocimiento, 7, 2. Disponible en: http://www.ibersid.eu/ojs/index. $\mathrm{php} / \mathrm{scire} /$ issue/archive

Illanas Duque, M. D.; Cabeza Llorca, A. (2005). La biblioteca complutense y nuevos recursos para el aprendizaje en campus virtuales: experiencias en otras universidades. En II Jornada Campus Virtual UCM: cómo integrar investigación y docencia en el CV-UCM, 2, 233-235. Disponible en: http://eprints.ucm.es/5777/1/Illanas_Duque.pdf

López Hernández. F. (2008). El campus de la Universidad Carlos III en Second Life.

El profesional de la información, 17, 6, 657-661.

Mackey, T. P.; Ho, J. (2008). Exploring the relationships between Web usability and students' perceived learning in Web-based multimedia (WBMM) tutorials. Computers \& Education, 50, 1, 386-409. Disponible en: http://www.sciencedirect.com/science/article/B6VCJ4M3J0MN-1/2/440298fa2f5e68dc37a1be73b07319d5

Mastromatteo, J. (2008). Tutoriales animados y podcasts, recursos Web 2.0 para la Alfabetización Informacional (ALFIN). En IX Congreso Iberoamericano de Informática Educativa, Caracas (Venezuela), 9. http://eprints.rclis.org/14973/

Ming-der Wu ; Shih-chuan Chen. (2008). Elementary schoolteachers' use of instructional materials on the web. The Electronic Library, 26, 6, 833-843. Disponible en: http:// ejournals.ebsco.com/direct.asp?ArticleID=4462810C50E9FE634152

Nancy H. Dewald, (1999). Transporting Good Library Instruction Practices into the Web Environment: an Analysis of Online Tutorials. The Journal of Academic Librarianship 25, $1,26-32$.

Pasch, G. (2001). La biblioteca digital soporte de la educación a distancia. Bibliotecas Digitales. Colima: Universidad de Colima, 151-158. http://www.ucol.mx/interfaces/interfaces2001/ ponencias/ponencias/Sp02.pdf

Poveda, A. Construir lecciones virtuales con Exelearning. Tutorial del Servicio de Bibliotecas de la Universidad de Salamanca http://web.usal.es/ angelpoveda/web\%20biologia/tutoriales/ Construir\%20lecciones\%20virtuales\%20con\%20Exelearning/index.html

Sastre Miralles, N. (2000). Productos y servicios para la formación de usuarios de bibliotecas universitarias: el uso de bases de datos en entornos Web. En VII Jornadas Españolas de Documentación, 7, 411-418.

Silver, S. L. and Nickel, L. T. (2005). Are online tutorials effective? A comparison of online and classroom library instruction methods. Research Strategies, 20, 4, 389-396. Disponible en: http://www.sciencedirect.com/science/article/B6W60-4MYMNX8-2/2/9c9a1d8b56ec 5441 b4d3bd36ca53384b

Smith, N. M.; Presser, P. (2005). Embed With the Faculty: Legal Information Skills Online. Journal of Academic Librarianship, 31, 3. Disponible en: http://www.sciencedirect.com/ science/journal/00991333

Somoza Fernández, M. (2009). Análisis de los tutoriales web creados por bibliotecas universitarias. Tesis Doctorals en Xarxa (TDX). Tesis Doctoral. Universidad de Barcelona. Departamento de Biblioteconomía y documentación. Disponible en: http://www.tesisenxarxa.net/TESIS_UB/ AVAILABLE/TDX-0401109-122232//MSF_TESIS.pdf 
Somoza-Fernández, M.; Abadal, E. (2009). Analysis of web-based tutorials created by academic libraries». The Journal of Academic Librarianship, 35, 2, 126-131. Disponible en: http:// www.sciencedirect.com/science/article/B6W50-4VNCC35-3/2/c382ba78e78da8296a462 $6 \mathrm{cb} 094 \mathrm{a} 23 \mathrm{aa}$

Tancheva, K. (2003). Online Tutorials for Library Instruction: An Ongoing Project Under Constant Revision. En ACRL Eleventh National Conference: Learning to Make a Difference. Disponible en: http://www.ala.org/ala/mgrps/divs/acrl/events/pdf/tancheva.PDF

Vives-Gràcia, J. (2004). Formación en el uso de la información en las bibliotecas de la Universitat Politècnica de Catalunya. Boletín de la ANABAD, 54, 1-2, 363-376. Disponible en: http:// dialnet.unirioja.es/servlet/fichero_articulo?articulo=1198721\&orden $=61517$ 